\title{
Neutral hydrogen in early-type dwarf galaxies of the Sculptor Group
}

\author{
Antoine Bouchard ${ }^{1,2}$, Gary DaCosta ${ }^{1}$, Helmut Jerjen ${ }^{1}$ \\ and Jürgen $\mathrm{Ott}^{2}$ \\ ${ }^{1}$ Research School of Astronomy \& Astrophysics, Mount Stromlo Observatory, Cotter Road, \\ Weston Creek, ACT 2611, Australia \\ ${ }^{2}$ Australia Telescope National Facility, CSIRO, PO Box 76, Epping NSW 1710, Australia
}

\section{Dwarf galaxy evolution and the morphology-density relation}

Dwarf galaxies are at the center of a long debated galaxy evolution puzzle. In the Local Group, there seems to be evidence that the exact morphology of a dwarf is strongly correlated with its environment: late-types (dwarf irregulars, dIrr) are further away from major group members than early-types (dwarf ellipticals and dwarf spheroidals, dE). In general, the early-types, with their smooth ellipsoid light distribution, have no detected interstellar medium (ISM), possess low angular momentum and have low current star formation rates. The late-types have an irregular appearance, large quantities of neutral hydrogen (HI), ongoing star formation and higher angular momentum.

The relation between the morphology of a dwarf and the number density of galaxies around it, the so-called morphology-density relation, and the apparent continuous range in dwarf galaxy properties suggests a possible evolutionary connection between the main types, with mixed type (dIrr/dE) galaxies forming the intermediate class. Grebel, Gallagher, \& Harbeck (2003) argue against this evolutionary scenario stating two major points: first the fading of a dIrr to the typical luminosity of a dE would produce an object with too low metallicity than what is observed and, second, the removal of the ISM from a dIrr would not produce a non-rotating dE.

The problem with the morphological classification is that it merely describes the behavior of the light distribution around galaxies and does not convey any physical information on the objects it classifies. Studies of the Local Group helped establish empirical relations between morphology and other physical parameters, but have failed to establish a direct quantitative correlation based on solid physical principles.

Consequently, if one wishes to understand dwarf galaxy evolution, a quantitative measure of the environmental impact needs to be established and related to the fundamental properties of galaxies (stellar population, etc.). High density environments maximize the effects of external gas removal mechanisms (e.g. ram pressure, tidal stirring) while a low density environment will minimize such factors, thereby enabling us to differentiate between an environmentally or internally generated effects.

In order to get such a quantitative measure of the environmental impact on the evolution of dwarfs in a low density setting, we have observed five galaxies of the Sculptor group: the dS0/Im ESO294-G010, the two dE/Im ESO540-G030 and ESO540-G032, the dSph ESO410-G005, and the dE Scl-dE1. In the cases of ESO294-G010, ESO540-G030, ESO540-G032, and ESO410-G005, photometric studies seem to indicate the presence of blue stars in the central regions of these galaxies (Karachentsev et al. 2000; Jerjen \& Rejkuba 2001; Karachentsev et al. 2002; Karachentsev et al. 2003) although in some cases these may be the result of photometric errors or foreground contamination. In addition 


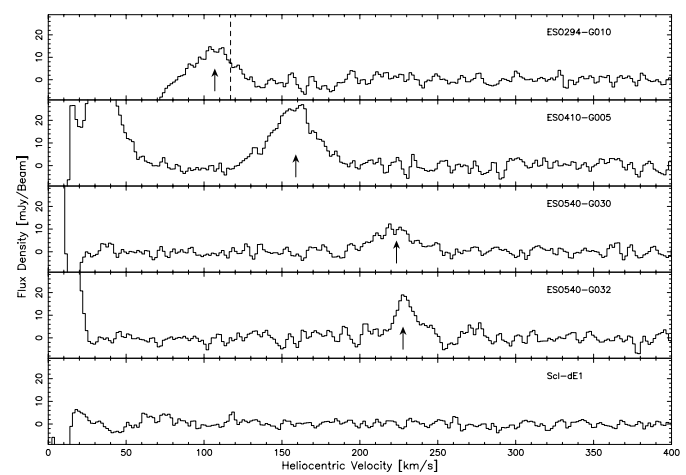

Figure 1. Parkes Hi spectra of the 5 observed dwarfs. In the top panel the vertical dashed line shows the optical velocity of the dwarf. The strong features around the $0 \mathrm{~km} \mathrm{~s}^{-1}$ velocity are artifacts from the Galactic Hi emission.

to these maybe young blue stars, Jerjen, Freeman \& Binggeli (1998) identified a small HII region in ESO294-G010.

The Parkes single dish radio-telescope was used to obtain deep high velocity resolution Hi spectra for the five targeted galaxies (Figure 1). The $8 \mathrm{MHz}$ bandpass gave a velocity coverage from -300 to $1300 \mathrm{~km} \mathrm{~s}^{-1}$, divided in 1024 channels, each $1.6 \mathrm{~km} \mathrm{~s}^{-1}$ wide.

\section{Results}

Figure 1 shows clear detections of four out of five targets. The objects have $\mathrm{M}_{H I}=$ $(2.4 \pm 0.3) \times 10^{5} \mathrm{M}_{\odot}$ for ESO294-G010 (D = 1.7 Mpc), $(7 \pm 2) \times 10^{5} \mathrm{M}_{\odot}$ for ESO410-G005 $(\mathrm{D}=1.9 \mathrm{Mpc}),(8 \pm 1) \times 10^{5} \mathrm{M}_{\odot}$ for ESO540-G030 $(\mathrm{D}=3.2 \mathrm{Mpc}),(4 \pm 1) \times 10^{5} \mathrm{M}_{\odot}$ for ESO540-G032 (D = 2.2 Mpc), and $\mathrm{M}_{H I}<2 \times 10^{5} \mathrm{M}_{\odot}$ for Scl-dE1 $(3 \sigma, \mathrm{D}=4.2 \mathrm{Mpc}$, assumes a velocity dispersion of $20 \mathrm{~km} \mathrm{~s}^{-1}$ ).

In the low density environment of the Sculptor group, most galaxies can be considered as essentially isolated objects. In this case, galaxy interactions will be few, making tidal stirring, disruption and ram pressure stripping very ineffective.

Comparing the case of the dSph ESO410-G005 and of the dE Scl-dE1 is extremely interesting. The former seems to contain some young blue stars (Karachentsev et al. $2000)$ and has significant amount of $\mathrm{HI}\left(\mathrm{M}_{H I} / \mathrm{L}_{B}=0.13\right)$ but is also closer $(230 \mathrm{kpc})$ to its nearest neighbour, NGC55 than Scl-dE1 is to NGC253 (460 kpc). The latter contains no detected $\mathrm{HI}\left(\mathrm{M}_{H I} / \mathrm{L}_{B}<0.08\right)$ and has a predominantly old stellar population. This emphasises that the morphology-density relation is not always applicable in particular cases. The isolated Local Group dE Tucana is another example of this phenomenon.

In conclusion, our findings do support the claim that "transition-type dwarfs are dSphs that kept their interstellar medium and therefore should replace dSph's in isolated locations where stripping is ineffective" (Grebel et al. 2003), but it fails to readily support a quantitative version of the morphology-density relation

\section{References}

Grebel, E.K., Gallagher, J.S. \& Harbeck, D. 2003, AJ 125, 1926

Jerjen, H., Freeman, K.C. \& Binggeli, B. 1998, AJ 116, 2873

Jerjen, H. \& Rejkuba, M. 2001, A\& $A 371,487$

Karachentsev, I.D. et al. 2000, ApJ 542, 128

Karachentsev, I.D. et al. 2002, $A \xi A 389,812$

Karachentsev, I.D. et al. 2003, A\&ऽ A 404, 93 